A - Research concept and design

B - Collection and/or assembly of data

$\mathrm{C}$ - Data analysis and interpretation

D - Writing the article

E - Critical revision of the article

F - Final approval of article

\section{An eight-week pulsed electromagnetic field improves physical functional performance and ankle-brachial index in men with Fontaine stage II peripheral artery disease}

\author{
Ahmad Mahdi Ahmad*1,A,C-F (D), Reham Raafat Mahmoud $2, A-B$ \\ ${ }^{1}$ Department of Physical Therapy for Cardiovascular and Respiratory \\ Disorders, Faculty of Physical Therapy, Cairo University, Giza, Egypt \\ ${ }^{2}$ Department of Physical Therapy, Alexandria Police Hospital, Alexandria, \\ Egypt
}

*Correspondence: Ahmad Mahdi Ahmad, Department of Physical Therapy for Cardiovascular and Respiratory Disorders, Faculty of Physical Therapy, Cairo University, Giza; email: Ahmed.mahdy@pt.cu.edu.eg

\title{
Abstract
}

Introduction: Peripheral artery disease (PAD) is a highly prevalent but insufficiently studied cardiovascular disease. Apart from exercise training, little evidence supports the use of complementary therapies such as pulsed electromagnetic field (PEMF) in PAD management despite its favorable cardiovascular effects. Therefore, this study aimed to assess effects of PEMF on treadmill walking performance and ankle-brachial index (ABI) in men with PAD.

Material and methods: Thirty male patients aged from 45 to 65 years with Fontaine stage II PAD were assigned to two groups; an experimental and a control group. Exclusion criteria were: Fontaine stage I, III and IV PAD; contraindications to exposure to electromagnetic fields; coronary artery, heart or chest disease; and orthopedic/neurological limitations to treadmill exercise test. Both groups received pharmacological treatment and dietary instructions, but only the experimental group received an eight-week PEMF (frequency: $15 \mathrm{~Hz}$; intensity: 20 Gauss; duration: 60 minutes) three sessions per week. A treadmill graded exercise test (GXT) was performed at baseline and post-intervention. The primary outcomes were walking parameters derived from the GXT (i.e., peak walk distance/walk time and pain-free walk distance/walk time), and the secondary outcome was the ABI.

Results: The experimental group showed significant improvements in the GXT outcomes compared to baseline and controls $(p<0.01)$, and a significant increase in the ABI compared to baseline $(p<0.01)$ that approached significance compared to the controls $(\mathrm{p}=0.012)$.

Conclusions: PEMF therapy could be suggested as a beneficially rehabilitative intervention, complementary to pharmacological treatment and dietary advice, that could help improve physical functional performance and ABI in men with Fontaine stage II PAD.

Keywords: peripheral artery disease, ankle-brachial index, graded exercise test, pulsed electromagnetic field, physical functional performance

\section{Introduction}

Peripheral artery disease (PAD) is one of the major cardiovascular diseases and represents the clinical presentation of atherosclerosis in the arteries of the lower limbs [1]. The global numbers of PAD cases and deaths have risen constantly each year from 1990 to 2019 , nevertheless, PAD is inadequately recognized

This is an Open Access journal, all articles are distributed under the terms of the Creative Commons Attribution-NonCommercial-ShareAlike 4.0 International (CC BY-NC-SA 4.0). License (http://creativecommons. org/licenses/by-nc-sa/4.0/). 
compared to other cardiovascular diseases and insufficiently studied [2]. Intermittent claudication is a common symptom in PAD characterized by cramps or pain in the muscles of the lower extremities during walking or exercise and is associated with a marked reduction in walking ability and/or physical functional performance [3]. Walking exercise is strongly recommended by almost all national and international guidelines as the first-choice lifestyle therapy for patients with PAD and intermittent claudication [4]. However, it is not uncommon that patients with PAD could have limited walking ability because of the severity of the intermittent claudication itself or other comorbid health concerns hindering them from adherence to walking exercises, therefore, alternate forms of therapy should be investigated for effectiveness in PAD.

The pulsed electromagnetic field (PEMF) has recently gained attention by researchers involved in the realm of cardiovascular disease management, owing to its multiple therapeutic benefits. There has been considerable evidence supporting the cardiovascular effects of PEMF in terms of stimulating angiogenesis and preserving cardiac function in rat models with ischemic lesions [5,6], improving flow-mediated dilatation and blood pressure in hypertensive subjects [7], increasing nitric oxide bioavailability, and reducing blood pressure in metabolic syndrome [8], as well as reducing the intimal medial thickness, increasing blood flow velocity, and improving the ankle-brachial index $(\mathrm{ABI})$ in patients with PAD $[9,10]$. Yet, there is insufficient evidence to support the functional outcomes of PEMF in terms of enhanced walking/physical functional performance in patients with PAD. One recent study [10] has investigated the effects of PEMF in combination with calf muscle exercises on maximum walking distance and maximal walk time in PAD patients and has found significant improvements in these variables.

Therefore, in order to further clarify the functional outcomes of PEMF in PAD, the present study was conducted to assess the effects of PEMF, in combination with pharmacological treatment and dietary instructions, on the walking parameters derived from treadmill graded exercise test (i.e., peak and pain-free walk distance/walk time) as primary outcomes and the ABI as a secondary outcome, compared to pharmacological treatment and dietary advice alone in male patients with PAD and intermittent claudication. The results of this study may aid efforts seeking a rehabilitative intervention alternative to exercising and complementary to pharmacological treatment for men with PAD and intermittent claudication.

\section{Material and methods}

This study was approved by the Ethics Committee of Human Scientific Research with an approval number of P.T.REC/012/002122. The Guidelines of the Declaration of Helsinki on the conduct of human research have been followed. Written informed consent was obtained from all participants.

\section{Sample size calculation}

The sample size was calculated based on an earlier study [10], for the main outcome of the present study [i.e., the peak walk distance (PWD)]. The power of the present study was set at $90 \%$ and the level of significance was set at a $p$-value of $<0.01$. The following formula was used for sample size detection: Sample size (n) $=2 \mathrm{SD}^{2}\left(Z_{\alpha / 2}+Z_{\beta}\right)^{2} / \mathrm{d}^{2},[11,12]$. Where: $Z_{\alpha / 2}=2.58$ for 2 tailed results at $\mathrm{p}<0.01 ; Z_{\beta}=1.28$ for a power of $90 \% ; \mathrm{SD}=$ Standard Deviation (estimated largest SD of the PWD) $=48.67$ (meters) [10]; $d=$ expected effect size $=$ average change of PWD in the study group - average change of PWD in the control group $=127.8-48.33=$ 79.47 (meters) [10]. Accordingly, sample size (n) $=2$ $(48.67)^{2} \times(2.58+1.28)^{2} \div(79.47)^{2}=11.17$. To allow for a $20-30 \%$ drop-out rate, four patients were also needed, and the total sample size was calculated to be 15 patients per group.

\section{Participants}

Thirty male patients with PAD were recruited by referral from a vascular specialist. The inclusion criteria were: diagnosis of PAD confirmed by an ABI at rest of $<0.9$, Fontaine stage II PAD (a history of leg pain), male patients, body mass index from 20 to 30 $\mathrm{kg} / \mathrm{m}^{2}$, and age from 45 to 65 years. Exclusion criteria were: asymptomatic PAD, current smoking, resting leg pain, acute/critical limb ischemia, ischemic trophic lesions, intermittent claudication of neurological or musculoskeletal origin, previous vascular surgery, uncontrolled hypertension, uncontrolled diabetes, contraindications for PEMF therapy (e.g., malignancy, soft tissue or skin infection, or bleeding disorders), contraindications for graded exercise test, and/or limitation in exercise tolerance by factors other than leg pain (e.g., coronary artery disease, congestive heart failure, chest disease, or back/joint problems). The participants were assigned by consecutive sampling into two equal groups; an experimental and a control group. Both groups received the pharmacological treatment for PAD and dietary instructions, whilst, the experimental group additionally received the PEMF therapy. 


\section{Measurements}

The age, the claudication history, and the co-morbid conditions of patients were reported during history taking. The body weight and height were measured at the baseline by a digital weight/height scale (BYH01, Health digital scale, made in china). The body mass index (BMI) of each patient was calculated as follows: $\mathrm{BMI}=$ body weight in kilograms/ body height in meters squared.

The Ankle-brachial index (ABI) was measured and interpreted by an ultrasonography specialist according to Aboyans et al. [13]. The ABI measurements were conducted at the baseline for patient selection and after 8 weeks for follow-up using a Doppler device (siemens acuson x300 ultrasound system, made in Germany). The systolic pressure of the right brachial artery, the right posterior tibial, and the right dorsalis pedis artery was measured. Then, the higher pressure value between the posterior tibial and the dorsalis pedis artery was used and divided by the systolic arm pressure to calculate the right $\mathrm{ABI}$. The same procedure was done for the left side. The ABI values measured for both sides were recorded and the lower value on either side was considered as the overall ABI, as reported by a previous study [14].

A treadmill graded exercise test (GXT) was conducted and supervised by a physiotherapist at baseline and after 8 weeks using an electrically automated treadmill (Weslo Cadence 1005, Model NO.WLTL 39093, made in the USA). The treadmill used in the GXT had a digital screen with multiple set buttons to adjust the speed and the inclination of the treadmill during the test. The digital screen also showed the time elapsed and the distance covered throughout the test. According to Hiatt et al. [15], the patients were instructed to begin the test by walking on the treadmill at a speed of $3.2 \mathrm{~km} /$ hour on a horizontal level ( $0 \%$ inclination) for 3 minutes. Afterward, the inclination of the treadmill was increased progressively by $3.5 \%$ every 3 minutes while keeping the speed constant at $3.2 \mathrm{~km} /$ hour. Throughout the test, the patients were instructed to continue walking on the treadmill till reaching the maximal claudication pain, the point at which the test had to be ended. Outcomes derived from GXT:

- Peak walk distance (PWD) - the longest distance recorded at the end of the test at which the patient was unable to proceed forward in the test because of severe claudication pain [16].

- Pain-free walk distance - the distance recorded once the patient first experienced claudication pain [16].

- Peak walk time (PWT) - the time elapsed from the beginning of the test to the point at which the patient stopped the test because of maximal claudication pain.
- Pain-free walk time - the time elapsed from the start of the test to the point at which the patient first reported the claudication pain.

\section{Interventions}

Pulsed electromagnetic field (PEMF) therapy

The PEMF therapy was administered by a physiotherapist and was introduced only to the patients in the experimental group using a magneto-therapy unit (BTL-5000 Series, made in the UK). The unit was set to apply rectangular pulses of a magnetic field ( $4.5 \mu$ $\mathrm{sec})$ at a low frequency of $15 \mathrm{HZ}$ and a very low intensity of 20 Gauss [9], through a solenoid $70 \mathrm{~cm}$ long adjusted over the legs and feet of both lower extremities whilst the patient in the supine position. The rising time from 0 to 20 Gauss was 200 milliseconds and the return time to zero was 24 milliseconds. The PEMF therapy was applied for 60 minutes/session [17], three sessions/ week, for a total of 8 weeks $[9,10]$.

\section{Pharmacological treatment}

A vascular specialist prescribed the drug therapy to all patients in both groups. Medications prescribed included anti-platelet medications (plavix oral), cholesterol-lowering medications (lipitor), medications to treat intermittent claudication (cilostazol), L-carnitine and omega 3 supplementation, anti-hypertensive medications (ACE inhibitors) for hypertensive patients, and anti-diabetic medications (metformin) for diabetics. The medications were similar in both groups.

\section{Dietary instructions}

Patients in both groups were instructed to reduce consumption of atherogenic foods (such as saturated/ animal fats and cholesterol), fried or processed food/ meat, salty food, or sugar-sweetened beverages; and to increase intake of healthy foods such as unsaturated fats and omega 3 fatty acids, fat-free protein, legumes, whole grains (instead of refined grains), vegetables and fruits (fibers, vitamins \& minerals rich sources). A follow-up was made every two weeks throughout the study period to assess the adherence of patients in both groups to the dietary instructions.

\section{Statistical analysis}

The Social Science Statistics software was used for statistical analysis. The Shapiro-Wilk test was used to assess the normality of data, and the Bartlett's test was used to assess the assumption of variance equality across the samples. Square root transformation was needed for some data to ensure equal variances before conducting ANOVA tests. The paired t-test was used to assess the changes in the mean values of the variables within each group. The one-way ANOVA test 
was used to assess the differences in the mean values of the variables between the two groups pre- and postintervention. Values of $p<0.01$ were considered statistically significant.

\section{Results}

At baseline, there were non-significant differences in age, anthropometric characteristics, and outcome measures between the two groups ( $p>0.01$ ), as shown in Table 1. In the experimental group, the GXT outcomes [i.e., the peak walk distance (PWD), pain-free walk distance, peak walk time (PWT), and pain-free walk time] and the ABI were significantly improved compared to baseline $(\mathrm{p}<0.01)$, as shown in Table 1 . In the control group, only the GXT outcomes were significantly improved compared to baseline $(\mathrm{p}<0.01)$, as shown in Table 1. Comparing the two groups revealed that the PWD, pain-free walk distance, PWT, and painfree walk time improved significantly in the experimental group compared to the control group $(\mathrm{p}<0.01)$, as shown in Table 1. For the ABI, the improvement was greater in the experimental group compared to the control group, but was not quite significant at $\mathrm{p}<0.01$ (i.e., $\mathrm{p}=0.012$ ), as shown in Table 1 .

The percent changes from baseline in the outcome measures in the two groups can be shown in Figure 1.

Tab. 1. Baseline characteristics and outcome measures in the two groups before and after the interventions

\begin{tabular}{|c|c|c|c|c|}
\hline \multicolumn{2}{|l|}{ Variables } & $\begin{array}{l}\text { Experimental group } \\
\qquad(\mathrm{n}=15)\end{array}$ & $\begin{array}{l}\text { Control group } \\
(\mathrm{n}=15)\end{array}$ & $\begin{array}{l}\text { Experimental vs. control group } \\
\text { p-value }\end{array}$ \\
\hline \multicolumn{5}{|c|}{ Baseline characteristics } \\
\hline \multicolumn{2}{|l|}{ Age (years) } & $56.4 \pm 5.55$ & $55.46 \pm 5.15$ & 0.636 \\
\hline \multicolumn{2}{|l|}{ Body weight (kg) } & $76.88 \pm 5.10$ & $80.33 \pm 5.09$ & 0.074 \\
\hline \multicolumn{2}{|l|}{ BMI $\left(\mathrm{kg} / \mathrm{m}^{2}\right)$} & $25.69 \pm 1.98$ & $26.91 \pm 1.88$ & 0.096 \\
\hline \multicolumn{5}{|l|}{ Outcome measures } \\
\hline \multirow{3}{*}{$\begin{array}{l}\text { Peak walk distance } \\
\text { (meters) }\end{array}$} & baseline & $265.2 \pm 38.5$ & $260.9 \pm 33.7$ & 0.749 \\
\hline & post & $432.9 \pm 45.2$ & $299.4 \pm 34.1$ & $<0.001^{*}$ \\
\hline & $\mathrm{p}$-value ${ }^{\mathrm{b}}$ & $<0.001^{*}$ & $<0.001^{*}$ & \\
\hline \multirow{3}{*}{$\begin{array}{l}\text { Pain-free walk } \\
\text { distance (meters) }\end{array}$} & baseline & $162.9 \pm 44.9$ & $158.8 \pm 34.5$ & 0.783 \\
\hline & post & $244.4 \pm 45.2$ & $164.3 \pm 33.5$ & $<0.001^{*}$ \\
\hline & $p$-value ${ }^{b}$ & $<0.001^{*}$ & $<0.001^{*}$ & \\
\hline \multirow{3}{*}{$\begin{array}{l}\text { Peak walk time } \\
\text { (minutes) }\end{array}$} & baseline & $8.54 \pm 0.97$ & $8.59 \pm 0.89$ & 0.876 \\
\hline & post & $15.04 \pm 1.03$ & $10.06 \pm 0.81$ & $<0.001^{*}$ \\
\hline & p-value ${ }^{b}$ & $<0.001^{*}$ & $<0.001^{*}$ & \\
\hline \multirow{3}{*}{$\begin{array}{l}\text { Pain-free walk time } \\
\text { (minutes) }\end{array}$} & baseline & $4.87 \pm 1.12$ & $5.17 \pm 0.95$ & 0.428 \\
\hline & post & $7.48 \pm 0.94$ & $5.53 \pm 0.94$ & $<0.001^{*}$ \\
\hline & $p$-value ${ }^{b}$ & $<0.001^{*}$ & $<0.001^{*}$ & \\
\hline \multirow[t]{3}{*}{ Overall ABI } & baseline & $0.70 \pm 0.06$ & $0.71 \pm 0.04$ & 0.740 \\
\hline & post & $0.77 \pm 0.06$ & $0.72 \pm 0.04$ & 0.012 \\
\hline & $p$-value ${ }^{b}$ & $<0.001^{*}$ & 0.029 & \\
\hline
\end{tabular}

Data are expressed as Means \pm SD. ${ }^{\text {a }}$ p-value based on the one-way ANOVA test; ${ }^{b}$ p-value based on the paired t-test; *Significant $\mathrm{p}$ value $(\mathrm{p}<0.01)$. BMI: Body mass index; ABI: ankle-brachial index. 


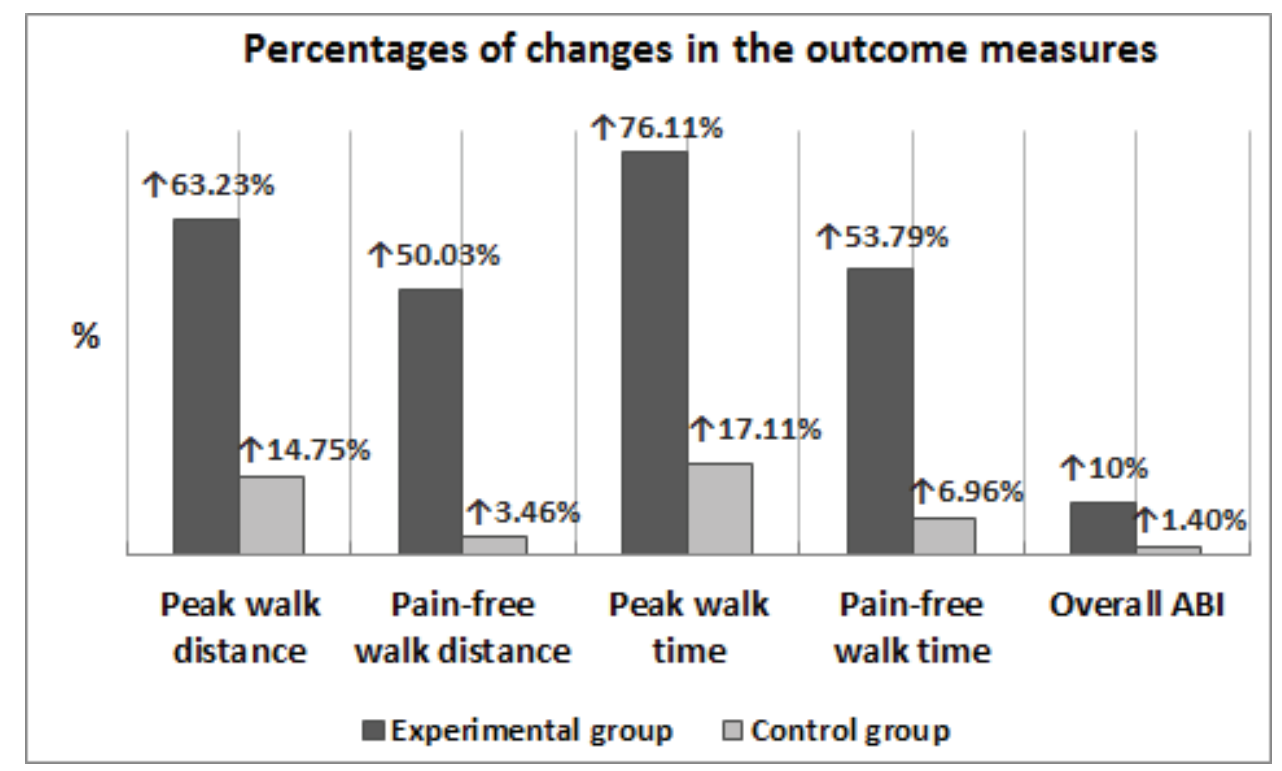

Fig. 1. Percent changes from baseline in the outcome measures in the two groups

\section{Discussion}

Pulsed electromagnetic field (PEMF) therapy has recently been the focus of cardiovascular research in different patient populations and has proved effective for several clinical outcomes. However, the functional outcomes of PEMF have been insufficiently studied in patients with peripheral arterial disease. The major findings of the present study can be summarized as follows: (a) There were significant improvements in the peak walk distance, pain-free walk distance, peak walk time, and pain-free walk time in the experimental group compared to baseline and the control group $(p<0.01)$. (b) Also, the improvement in the ABI was significant in the experimental group compared to baseline $(p<0.01)$ and approached significance $(p=0.012)$ compared to the control group.

Similar to these findings, Mohamed et al. [10] reported that PEMF combined with heel raising exercise induced significant increases in the maximal walking distance and maximal walking time compared to the baseline and the controls in patients with PAD (Fontaine stage II). They also found that the ABI in the PEMF group was significantly increased compared to the baseline which was also consistent with the ABI finding reported in our experimental group. It should be mentioned that their effects could be owing to the combination of PEMF and calf muscle exercises and not to PEMF therapy alone. Worth noting, the PEMF therapy has induced favorable improvements in physical functional performance in other patient populations. For example, in older adults, PEMF has induced a greater increase in self-paced gait speed compared to the placebo group [18]. In Parkinson's disease patients, the freezing of gait, a sudden attack of immobility usually experienced during walking, has been alleviated following PEMF therapy [19]. Also, in patients with multiple sclerosis, PEMF has induced significant improvements in the disease-related fatigue symptoms $[20,21]$. Furthermore, PEMF has enhanced the physical function in patients with osteoarthritis [22] and reduced the physical disability in patients with low back pain [23]. Despite the discrepancy in patient populations and PEMF parameters, the results of the aforementioned studies [18-23] might be in line with the improvement in walking functional performance reported in our study following PEMF therapy.

An improved explanation of the pathophysiology of limb symptoms in PAD would lead to a better understanding of the mechanisms underlying the functional effects of PEMF therapy reported in the present study. The main pathophysiological occurrences in PAD underlying the intermittent claudication and the related limitations in walking functional performance can be summarized as [24]: (i) A reduction of blood flow through the obstructed arteries that leads to an imbalance between oxygen \& nutrients supply and the metabolic demands generated by the working muscles during ambulatory activities/walk exercises; (ii) Impaired angiogenesis and vascular dysfunction; (iii) Chronic inflammation caused by ischemic insults and metabolic abnormalities within the muscles. Multiple physiological effects of PEMF such as vasodilatory, angiogenic, and anti-inflammatory/analgesic effects [25-27], could explain the significant improvements in walking functional parameters and $\mathrm{ABI}$ following 
PEMF therapy. These effects in combination could alter the underlying pathophysiology responsible for walking disability in PAD, resulting in improved circulation to the ischemic muscles, enhanced recovery from inflammation, increased muscle work, and better walking functional performance. The underlying mechanism responsible for the vasodilatory effect of PEMF involves nitric oxide release [28,29], which is a potent vasodilator agent resulting in marked arteriolar vasodilatation [25]. The increase in the diameter of the blood vessels as a result of vasodilatation following PEMF exposure has been associated with an increase in blood flow velocity in patients with peripheral ischemia $[9,17]$. The mechanism underlying PEMF-mediated angiogenesis involves promoted activation, proliferation, migration, and reticular structure formation of vascular endothelial cells; as well as stimulated expression of vascular endothelial growth factor, fibroblast growth factor 2, and other angiogenic growth factors [26]. By increasing the diameter and the number of blood vessels supplying blood to the ischemic muscles, an improvement in muscle action occurs with a consequent improvement in walking tolerability. Further, the anti-inflammatory effect of PEMF could be explained by the modulation of the inflammatory processes via regulation of the secretion of the pro- and anti-inflammatory cytokine during different stages of inflammation, which could have beneficial effects in terms of enhanced regeneration and functional recovery of inflamed tissues [27]. PEMF has also been suggested to promote the resolution of chronic inflammation by mediating changes in cellular gene expression [30].

Interestingly, one of the theories explaining the mechanism of PEMF action on the circulation is the pearl chain effect theory, which assumes that PEMF generates a magnetic polarization of red and white blood cells causing them to move through the blood vessel in a pearl chain-like form or a single-file rather than randomly floating in the bloodstream. Thereby, the red and white blood cells would move in an efficient way inside the small arteries and arterioles with narrowed lumen, bringing more oxygen and nutrients to the ischemic tissue and removing wastes [31]. Another interesting theory suggested that every cell in the body has its own frequency with which the externally applied electromagnetic field has to resonate in order to be effective, and that if the electromagnetic field is applied with a higher or a lower frequency than the cell's frequency, no effects appear [32]. In other words, for optimum biophysical interaction between the electromagnetic signals and the biological tissues, both the frequency of the electromagnetic field and the frequency of the cell have to be similar. Notably, the biophysical response depends not only on the frequency of the external magnetic field but also on some other properties, such as the intensity, the waveform, and the duration of exposure. Thus, it could be assumed that the PEMF applied in the present study with the prescribed parameters, based on previous studies $[9,10,17]$, could have resonated in a harmonic way with the recipient cells resulting in an efficient biophysical interaction and favorable outcomes in the ischemic tissues.

Unsurprisingly, significant changes in the peak/painfree walk distance and peak/pain-free walk time were found in the control group compared to the baseline in the current study. This phenomenon has been previously reported and has been known to occur as a result of a learning effect of the graded treadmill exercise test [33]. The repeated practice of exercise test at the baseline and follow-up by PAD patients, even if they received no intervention, leads to improvement in their treadmill performance outcomes including peak/painfree walk distance \& walk time $[33,34]$. It should be pointed out that even though treadmill exercise training is the gold standard method of rehabilitation of PAD patients with intermittent claudication, high attrition rates from exercise programs have been reported as several patients could be unable to adhere to exercise because of many reasons such as exercise-induced severe claudication pain, poor physical functional performance, and/or presence of co-existing health issues [35] (e.g., cardiopulmonary, metabolic, musculoskeletal, or neurological). Obviously, the PEMF is a passive intervention that requires no effort from the patients and could therefore represent a more suitable form of therapy than traditional exercise therapy for PAD patients who have poor adherence to regular exercise due to health issues.

Finally, as with the majority of studies, the findings of the present study should be interpreted in light of some limitations. The non-random allocation of subjects with the potential of bias represents the main limitation in this study. Also, the lack of a placebo control group with the possibility of psychological conditioning represents another limitation. Further, only male patients with Fontaine stage II PAD were enrolled in the current study, which could limit the generalization of the results to other PAD patients with different characteristics. Nevertheless, the principal strength of this study lies in the newly investigated topic in the domain of complementary/alternative therapy for cardiovascular diseases and may guide other researchers in the future. Besides, sample size was calculated with a power of $90 \%$ and an alpha level of 0.01 for optimal detection of statistical significance and sound interpretation of the results. 


\section{Conclusions}

An eight-week pulsed electromagnetic field (PEMF) therapy in addition to pharmaceutical therapy and dietary advice could be a more effective treatment regime than pharmacological therapy and dietary advice alone for improving treadmill walking performance and $\mathrm{ABI}$ in male patients with symptomatic PAD. The PEMF, therefore, could be proposed as an effective intervention complementary to pharmacological therapy and dietary instructions in PAD management that could help induce further improvements in physical functional performance in men with Fontaine stage II PAD. Future studies are needed to verify the results of the present study.

\section{Acknowledgement}

The authors would like to express a very great appreciation to Dr. Janet McDowell, Advanced Respiratory Practitioner Physiotherapist, Belfast Health and Social Care Trust, for her valuable language check and proofreading. Her willingness to give effort and time so generously has been very much appreciated.

\section{Funding}

This research received no external funding.

\section{Conflict of interests}

The authors have no conflict of interest to declare.

\section{References}

1. Eid MA, Mehta KS, Goodney PP. Epidemiology of peripheral artery disease. Seminars in Vascular Surgery. 2021; 34(1): 38-46.

2. Roth GA, Mensah GA, Johnson CO, Addolorato G, Ammirati E, Baddour LM, et al. Global Burden of Cardiovascular Diseases and Risk Factors, 19902019: Update From the GBD 2019 Study.Journal of the American College of Cardiology. 2020; 76(25): 2982-3021.

3. Spannbauer A, Chwała M, Ridan T, Berwecki A, Mika P, Kulik A, et al. Intermittent Claudication in Physiotherapists' Practice. Biomed Res Int. 2019; 2019: 2470801.

4. Harwood AE, Pymer S, Ingle L, Doherty P, Chetter IC, Parmenter B, et al. Exercise training for intermittent claudication: a narrative review and summary of guidelines for practitioners. BMJ Open Sport Exerc Med. 2020; 6(1): e000897.

5. Peng L, Fu C, Liang Z, Zhang Q, Xiong F, Chen L, et al. Pulsed Electromagnetic Fields Increase Angiogenesis and Improve Cardiac Function After Myocardial Ischemia in Mice. Circ J. 2020; 84(2): 186-93.
6. Pan Y, Dong Y, Hou W, Ji Z, Zhi K, Yin Z, et al. Effects of PEMF on microcirculation and angiogenesis in a model of acute hindlimb ischemia in diabetic rats. Bioelectromagnetics. 2013; 34(3): 180-8.

7. Stewart GM, Wheatley-Guy CM, Johnson BD, Shen WK, Kim CH. Impact of pulsed electromagnetic field therapy on vascular function and blood pressure in hypertensive individuals. J Clin Hypertens (Greenwich). 2020; 22(6): 1083-9.

8. Kim CH, Wheatley-Guy CM, Stewart GM, Yeo D, Shen WK, Johnson BD. The impact of pulsed electromagnetic field therapy on blood pressure and circulating nitric oxide levels: a double blind, randomized study in subjects with metabolic syndrome. Blood Press. 2020; 29(1): 47-54.

9. Ayad P, Aziz A, Mohamed A, Shendy M. Effect of Pulsed Magnetic Field Therapy VersusAerobic Training on Peripheral Arteries in Type 2 Diabetes . Med. J. Cairo Univ. 2020; 88(5): 2269-75.

10. Mohamed MM, El-Nahas NG, Hamed M H, Louis NN. Interferential Electrical Stimulation Versus Pulsed Electro- Magnetic Field in Management of Intermittent Claudication. The Egyptian Journal of Hospital Medicine. 2020; 80: 654-61.

11. Charan J, Biswas T. How to calculate sample size for different study designs in medical research? Indian $\mathrm{J}$ Psychol Med. 2013; 35(2): 121-6.

12. Kadam P, Bhalerao S. Sample size calculation. Int J Ayurveda Res. 2010; 1(1): 55-7.

13. Aboyans V, Criqui MH, Abraham P, Allison MA, Creager MA, Diehm C, et al. Measurement and interpretation of the ankle-brachial index: a scientific statement from the American Heart Association. Circulation. 2012; 126(24): 2890-909.

14. Barone Gibbs B, Dobrosielski DA, Althouse AD, Stewart KJ. The effect of exercise training on anklebrachial index in type 2 diabetes. Atherosclerosis. 2013; 230(1): 125-30.

15. Hiatt WR, Nawaz D, Regensteiner JG, Hossack KF. The evaluation of exercise performance in patients with peripheral vascular disease. J Cardiopulm Rehabil. 1988; 8(12): 525-32.

16. Kruidenier LM, Nicolaï SP, Willigendael EM, de Bie RA, Prins MH, Teijing JAW. Functional claudication distance: a reliable and valid measurement to assess functional limitation in patients with intermittent claudication. BMC Cardiovasc Disord. 2009; 9: 9.

17. Kwan RL, Wong WC, Yip SL, Chan KL, Zheng YP, Cheing GL. Pulsed electromagnetic field therapy promotes healing and microcirculation of chronic diabetic foot ulcers: a pilot study. Adv Skin Wound Care. 2015; 28(5): 212-9.

18. Giusti A, Giovale M, Ponte M, Fratoni F, Tortorolo U, De Vincentiis A, et al. Short-term effect of low- 
intensity, pulsed, electromagnetic fields on gait characteristics in older adults with low bone mineral density: a pilot randomized-controlled trial. Geriatr Gerontol Int. 2013; 13(2): 393-7 .

19. Sandyk R. Freezing of gait in Parkinson's disease is improved by treatment with weak electromagnetic fields. Int J Neurosci. 1996; 85(1-2): 111-24.

20. Lappin MS, Lawrie FW, Richards TL, Kramer ED. Effects of a pulsed electromagnetic therapy on multiple sclerosis fatigue and quality of life: a double-blind, placebo controlled trial. Altern Ther Health Med. 2003; 9(4): 38-48.

21. Zielińska-Nowak E, Włodarczyk L, Kostka J, Miller E. New Strategies for Rehabilitation and Pharmacological Treatment of Fatigue Syndrome in Multiple Sclerosis. J Clin Med. 2020; 9(11): 3592.

22. Yang X, He H, Ye W, Perry TA, He C. Effects of Pulsed Electromagnetic Field Therapy on Pain, Stiffness, Physical Function, and Quality of Life in Patients With Osteoarthritis: A Systematic Review and MetaAnalysis of Randomized Placebo-Controlled Trials. Phys Ther. 2020; 100(7): 1118-31.

23. Andrade R, Duarte H, Pereira R, Lopes I, Pereira H, Rocha R, et al. Pulsed electromagnetic field therapy effectiveness in low back pain: A systematic review of randomized controlled trials. Porto Biomed J. 2016; 1(5): 156-63.

24. Hamburg NM, Creager MA. Pathophysiology of Intermittent Claudication in Peripheral Artery Disease. Circ J. 2017; 81(3): 281-9.

25. Smith TL, Wong-Gibbons D, Maultsby J. Microcirculatory effects of pulsed electromagnetic fields. J Orthop Res. 2004; 22(1): 80-4.

26. Peng L, Fu C, Wang L, Zhang Q, Liang Z, He C, et al. The Effect of Pulsed Electromagnetic Fields on Angiogenesis. Bioelectromagnetics. 2021; 42(3): 250-8.

27. Ross CL, Zhou Y, McCall CE, Soker S, Criswell TL. The Use of Pulsed Electromagnetic Field to Modulate Inflammation and Improve Tissue Regeneration: A Review. Bioelectricity. 2019; 1(4): 247-59.
28. McKay JC, Prato FS, Thomas AW. A literature review: the effects of magnetic field exposure on blood flow and blood vessels in the microvasculature. Bioelectromagnetics. 2007; 28(2): 81-98.

29. Pilla AA. Electromagnetic fields instantaneously modulate nitric oxide signaling in challenged biological systems. Biochem Biophys Res Commun. 2012; 426(3): 330-3.

30. Kubat NJ, Moffett J, Fray LM. Effect of pulsed electromagnetic field treatment on programmed resolution of inflammation pathway markers in human cells in culture. J Inflamm Res. 2015; 8: 59-69.

31. Sharon TA. Utilizing Pulsed Electromagnetic Field (PEMF) Therapy (Diapulse ${ }^{\circledR}$ ) for Drug-Free Pain Management to Eliminate Reliance on Opioids for Non-Malignant Chronic Pain: A literature Review and Proposal for Pilot Study. Clin J Nurs Care Pract. 2019; 3: 27-34.

32. Goodman EM, Greenebaum B, Marron MT. Effects of electromagnetic fields on molecules and cells. Int Rev Cytol. 1995; 158: 279-338.

33. Hiatt WR, Rogers RK, Brass EP. The treadmill is a better functional test than the 6-minute walk test in therapeutic trials of patients with peripheral artery disease. Circulation. 2014; 130(1): 69-78.

34. Treat-Jacobson D, McDermott MM, Bronas UG, Campia U, Collins TC, Criqui MH, et al. American Heart Association Council on Peripheral Vascular Disease; Council on Quality of Care and Outcomes Research; and Council on Cardiovascular and Stroke Nursing. Optimal Exercise Programs for Patients With Peripheral Artery Disease: A Scientific Statement From the American Heart Association. Circulation. 2019; 139(4): e10-e33.

35. Abaraogu U, Ezenwankwo E, Dall P, Tew G, Stuart $\mathrm{W}$, Brittenden J, et al. Barriers and enablers to walking in individuals with intermittent claudication: A systematic review to conceptualize a relevant and patientcentered program. PLoS One. 2018; 13(7): e0201095. 